\title{
Isometries of systolic spaces
}

by

\author{
Tomasz Elsner (Columbus, OH, and Wrocław)
}

\begin{abstract}
We provide a classification of isometries of systolic complexes corresponding to the classification of isometries of $\operatorname{CAT}(0)$-spaces. We prove that any isometry of a systolic complex either fixes the barycentre of some simplex (elliptic case) or stabilizes a thick geodesic (hyperbolic case). This leads to an alternative proof of the fact that finitely generated abelian subgroups of systolic groups are undistorted.
\end{abstract}

1. Introduction. A systolic complex is a simplicial analogue of a nonpositively curved metric space, introduced by T. Januszkiewicz and J. Świątkowski in [JS2] and independently by F. Haglund in [Ha]. It is a connected, simply connected simplicial complex satisfying some local combinatorial condition (see Definition 2.1) with many properties similar to properties of CAT(0)-spaces. However, systolicity does not imply, and is not implied by, nonpositive curvature of the complex equipped with the standard piecewise Euclidean metric, for which simplices are regular Euclidean simplices.

In this paper we provide a classification of individual isometries of systolic spaces, similar to the classification of isometries of nonpositively curved metric spaces. The first result of the paper is the following:

THEOREM 1.1 (see Theorem 3.5). If $g$ is a simplicial isometry of a uniformly locally finite systolic complex $X$ then either there is a g-invariant simplex of $X$ or there is a $g^{n}$-invariant geodesic in $X$ for some $n \geq 1$.

If an isometry stabilizes a simplex, then the barycentre of the simplex can be considered a fix-point, so isometries of this type will be called elliptic. The above theorem proves that some power of a nonelliptic isometry has an invariant geodesic on which it acts by translation (it is a hyperbolic isometry). Unfortunately, the following example shows that there are non-

2000 Mathematics Subject Classification: 20F65, 20F67.

Key words and phrases: systolic complex, minimal surface, elliptic isometry, hyperbolic isometry. 
elliptic isometries of systolic spaces which do not have an invariant geodesic (i.e. the power $n$ in Theorem 1.1 is essential).

Example 1.2. Let $k \geq 2$. Define a simplicial complex $A_{k}$ such that $A_{k}^{(0)}=\mathbb{Z}$ and $\sigma \subset A_{k}^{(0)}$ spans a simplex if and only if $\left|a-a^{\prime}\right| \leq k$ for all $a, a^{\prime} \in \sigma$. The complex $A_{k}$ is systolic (see Definition 2.1) and the isometry $\gamma_{n}: A_{k} \rightarrow A_{k}$ induced by the map $\mathbb{Z} \ni x \mapsto x+n \in \mathbb{Z}$ has no invariant geodesics if $n$ is not divisible by $k$.

However, the situation described in the example is the only possible case when there is no invariant geodesic - for any nonelliptic isometry of a systolic complex there is an invariant subcomplex isomorphic to $A_{k}$ on which the isometry acts as $\gamma_{n}$ in the example. This motivates introducing the concept of a thick axis (which is a subcomplex at Hausdorff distance at most 1 from a geodesic) and stating the elliptic/hyperbolic dichotomy for systolic spaces in the following way:

THEOREM 1.3 (see Theorem 3.8). If $g$ is a simplicial isometry of a uniformly locally finite systolic complex $X$ then either $g$ stabilizes the barycentre of some simplex (elliptic case) or there is a g-invariant thick axis, i.e. a full subcomplex $A \subset X$ isomorphic to $A_{k}$ for some $k \geq 1$ (hyperbolic case).

As a corollary we obtain a proof (alternative to [JS2]) of the fact that infinite cyclic subgroups of a group acting cocompactly and properly discontinuously on a systolic complex are undistorted (see Corollary 3.9).

The rest of the paper is devoted to the proof of Theorem 1.3.

2. Systolic complexes. If $X$ is a simplicial complex and $\sigma$ a simplex of $X$, then the link of $X$ at $\sigma$ is the subcomplex of $X$ consisting of all simplices that are disjoint from $\sigma$ and together with $\sigma$ span a simplex of $X$.

Any subcomplex $\gamma \subset X$ isomorphic to a triangulation of a circle is called a cycle in $X$. The length of $\gamma$ (denoted $|\gamma|$ ) is the number of its edges. A diagonal of a cycle is an edge joining its two nonconsecutive vertices.

A simplicial complex $X$ is flag if every finite set of its vertices pairwise connected by edges spans a simplex of $X$. A subcomplex $Y \subset X$ is full if every simplex of $X$ whose vertices are all contained in $Y$ is also contained in $Y$.

Whenever in the paper we refer to a metric on a simplicial complex, we think of the combinatorial metric (i.e. the geodesic metric in which every edge has length 1) only on the 1-skeleton of the complex - we do not introduce any metric on the whole complex. Similarly, we use the symbol $d_{X}$ to denote the combinatorial metric on $X^{(1)}$. Moreover, throughout the paper a geodesic in a simplicial complex $X$ means actually a geodesic in $X^{(1)}$ having both endpoints in $X^{(0)}$. 
Definition 2.1 (see [JS1]). A simplicial complex $X$ is called:

- 6-large if it is flag and every cycle $\gamma$ in $X$ of length $4 \leq|\gamma|<6$ has a diagonal;

- locally 6-large if the link at every simplex of $X$ is 6-large;

- systolic if it is locally 6-large, connected and simply connected.

By the following fact the local condition in the definition of systolicity can be replaced with the global one by replacing the words "locally 6-large" with "6-large".

FACT 2.2 ([JS2, Proposition 1.4]). Every systolic complex is 6-large. In particular, a cycle of length smaller than 6 in a systolic complex bounds a triangulated disc with no internal vertices.

Minimal surfaces. The main tool used in the paper is the theory of minimal surfaces in systolic complexes, developed in [E]. A minimal surface is a simplicial map $S: \triangle \rightarrow X$ from a triangulation $\triangle$ of a 2-disc to a systolic complex $X$ such that $\triangle$ has the minimal number of triangles among all maps extending $\left.S\right|_{\partial \triangle}$. The existence of minimal surfaces follows from the contractibility of systolic complexes ([JS2, Theorem 4.1]) and is stated as follows:

Lemma 2.3 ([E, Lemma 4.2]). Let $X$ be a systolic space. Then:

(1) Any simplicial map $f: S^{1} \rightarrow X$ where $S^{1}$ is a triangulated circle can be extended to a minimal surface (the extension need not be unique).

(2) For any minimal surface $S: \triangle \rightarrow X$ the domain $\triangle$ is a systolic complex.

For any vertex $v$ of a triangulation $\triangle$ of a disc we define the defect at $v$ by the following formula:

$$
\operatorname{def}(v)= \begin{cases}6-\# \text { triangles in } \triangle \text { containing } v\} & \text { if } v \notin \partial \triangle \\ 3-\# \text { triangles in } \triangle \text { containing } v\} & \text { if } v \in \partial \triangle\end{cases}
$$

We call vertices (non)positive, (non)negative or (non)zero if so are their defects. The term the sum of the defects of $\triangle$ along a boundary line $l \subset \partial \triangle$ will be used for the sum of the defects at all vertices on $l$ but the endpoints.

Lemma 2.4 (Gauss-Bonnet Lemma). If $\triangle$ is a triangulation of a 2-disc, then

$$
\sum_{v \in \triangle^{(0)}} \operatorname{def}(v)=6
$$

In particular, if $\triangle$ is a systolic triangulation (e.g. the domain of a minimal surface), then the sum of the defects at boundary vertices is at least 6 , with equality if and only if $\triangle$ has no internal vertices of negative defects. 
FACT 2.5. If $\triangle$ is a triangulation of a 2 -disc and $g$ is a geodesic in $\triangle$ contained in $\partial \triangle$, then the sum of the defects along $g$ is at most 1 .

Proof. Since $g$ is geodesic, there are no vertices of defect 2 on $g$ different from its endpoints, and if $g$ passes through vertices $u$ and $v$ of defect 1 , then there is at least one negative vertex on $g$ lying between $u$ and $v$. Thus any positive vertex on $g$ has defect 1 and any two positive vertices are separated by a negative one, so the sum of the defects is at most 1 .

The proof of Theorem 1.3 is based on the following two theorems, which summarize the relevant results from [E, Sections 3-5]:

TheOREM 2.6. Let $X$ be a systolic complex, $P$ a simplicial triangulation of a strip $\mathbb{R} \times I$, and $f: P \rightarrow X$ a simplicial map. Suppose that

(i) every vertex $v \in \partial P$ has defect $-1,0$ or 1 ;

(ii) every internal vertex $v \in P$ has defect 0 ;

(iii) in each boundary component of $P$, any two nonzero vertices of the same sign are separated by a vertex of the opposite sign;

(iv) $\partial P$ is a full subcomplex of $P$;

(v) for every internal vertex $v \in P$ and for every edge uw $\subset P$ with both endpoints at internal vertices, $\left.f\right|_{N(v)}$ and $\left.f\right|_{N(u w)}$ are minimal surfaces.

Then $f$ maps each boundary component of $\partial P$ to a geodesic in $X$.

Here and subsequently, $N(K)$ denotes the union of all closed simplices intersecting $K$.

Proof. The complex $P$ is an increasing union of simplicial discs $P_{n}$ such that any internal vertex $v \in P_{n}$ has defect 0 , any boundary vertex $v \in P_{n}$ has defect at least -1 and any two negative vertices on $\partial P_{n}$ are separated by a positive one. By [E, Lemma 3.5] the simplicial maps $\left.f\right|_{P_{n}}: P_{n} \rightarrow X$ satisfy the assumptions of [E, Theorem 4.12]. By [E, Proposition 4.7(2), Theorem 4.12 and Corollary 4.11(2)] the intersection of $P_{n}$ with any boundary component of $P$ is mapped by $f$ to a geodesic in $X$. Since $P$ is the increasing union of $P_{n}$ for $n=1,2, \ldots$, each boundary component of $P$ is mapped to a geodesic in $X$.

Theorem 2.7 (see [E, Theorem 5.2]). Let $\mathbb{E}_{\triangle}^{2}$ be a triangulation of the Euclidean plane by congruent equilateral triangles, $X$ a systolic complex and $F: \mathbb{E}_{\triangle}^{2} \rightarrow X$ a simplicial map. If $\left.F\right|_{N(v)}$ is an isometric embedding for any vertex $v \in \mathbb{E}_{\triangle}^{2}$ and $\operatorname{diam}(\operatorname{Im} F) \geq 3$, then $F$ is an isometric embedding.

3. Classification of isometries. In the theory of CAT(0)-spaces an elliptic isometry is an isometry with a fix-point. For systolic complexes we define an elliptic isometry to be a simplicial isometry that stabilizes some 
simplex, so it can also be thought of as an isometry with a fix-point (the barycentre of the simplex).

First we prove that any power of a nonelliptic isometry of a systolic complex is also nonelliptic. Thus, in the subsequent part of the paper we will only need to consider free actions of $\mathbb{Z}$ on systolic complexes.

Proposition 3.1. Let $g$ be a simplicial isometry of a systolic complex $X$. If there is a $g^{n}$-invariant simplex of $X$ for some $n \geq 1$, then there is a $g$-invariant simplex of $X$.

Proof. Let $\sigma$ be a $g^{n}$-invariant simplex of $X$. Then for a sufficiently large integer $R>0$ the full subcomplex

$$
Y:=\mathcal{N}_{R}(\sigma) \cap \mathcal{N}_{R}(g(\sigma)) \cap \cdots \cap \mathcal{N}_{R}\left(g^{n-1}(\sigma)\right) \subset X
$$

is nonempty and $g$-invariant. It is also geodesically convex, since by [HS, Corollary 4.10] balls around vertices in systolic complexes are geodesically convex. Hence $Y$ is itself a systolic complex, as a geodesically convex subcomplex of a systolic complex ([JS2, Lemma 7.2]; the notion of convexity used in [JS2] coincides by [HS, Proposition 4.9] with the notion of geodesic convexity), so $Y$ is contractible (by [JS2, Theorem 4.1] every systolic complex is contractible). Thus, $H_{i}(Y)=0$ for $i=1,2, \ldots$ and by the Lefschetz Fix-Point Theorem the isometry $\left.g\right|_{Y}: Y \rightarrow Y$ has a fix-point $y \in Y$. Since $g$ preserves the simplicial structure, the minimal simplex of $Y$ containing $y$ is $g$-invariant.

3.1. The minimal displacement. For a simplicial isometry $g$ of a simplicial complex $X$ we define the minimal displacement to be

$$
|g|=\min _{x \in X^{(0)}} d_{X}(x, g(x)) .
$$

We denote by $\operatorname{Min}(g)$ the full subcomplex of $X$ spanned by all vertices $v \in X$ satisfying $d_{X}(v, g(v))=|g|$. The subcomplex $\operatorname{Min}(g)$ is clearly nonempty. Moreover, in the case where $g$ is nonelliptic it is a systolic complex whose 1skeleton is isometrically embedded in $X$ (see Propositions 3.3 and 3.4 below).

FACT 3.2. Let $g$ be a nonelliptic simplicial isometry of a systolic complex $X$. Then for any vertex $v \in \operatorname{Min}(g)$ and any geodesic $\alpha \subset X^{(1)}$ joining $v$ to $g(v)$ the simplicial path $\gamma: \mathbb{R} \rightarrow X$ (where $\mathbb{R}$ is equipped with the simplicial structure with $\mathbb{Z}$ as the set of vertices) defined to be the concatenation of the geodesics $g^{n}(\alpha), n \in \mathbb{Z}$, is a $|g|$-geodesic, i.e.

$$
d(\gamma(a), \gamma(b))=|a-b| \quad \text { if } a, b \text { are integers such that }|a-b| \leq|g| .
$$

In particular, $\operatorname{Im}(\gamma) \subset \operatorname{Min}(g)$.

Proof. We prove the statement for the case where $|a-b|=|g|$ (this implies the general case). Then, by the construction of $\gamma$, either $\gamma(b)=$ 
$g(\gamma(a))$ or $\gamma(a)=g(\gamma(b))$, thus we have $d(\gamma(a), \gamma(b)) \geq|g|$. The opposite inequality follows from the fact that $\gamma$ is a simplicial map.

Proposition 3.3. If $g$ is a nonelliptic simplicial isometry of a systolic complex $X$, then the 1-skeleton of $\operatorname{Min}(g)$ is isometrically embedded in $X$.

Proof. Suppose the 1-skeleton of $\operatorname{Min}(g)$ is not isometrically embedded. Then there exist vertices $v, w \in \operatorname{Min}(g)$ such that no geodesic in $X$ with endpoints $v$ and $w$ is contained in $\operatorname{Min}(g)$. Choose $v$ and $w$ so that $d_{X}(v, w)$ is minimal (clearly, $d_{X}(v, w)>1$ ). Let geodesics $\alpha, \beta$ and $\xi$ join $v$ to $g(v)$, $w$ to $g(w)$ and $v$ to $w$, respectively. Then $g(v)$ is joined to $g(w)$ by $g(\xi)$, and

$$
\xi \cap \operatorname{Min}(g)=\{v, w\}, \quad g(\xi) \cap \operatorname{Min}(g)=\{g(v), g(w)\}
$$

by the minimality of $d_{X}(v, w)$, and $\alpha, \beta \subset \operatorname{Min}(g)$ by Fact 3.2. Thus, the geodesics $\alpha$ and $\beta$ intersect the geodesics $\xi$ and $g(\xi)$ only at the endpoints.

Suppose there is a vertex $x \in \xi \cap g(\xi)$. Then $g(x) \in g(\xi)$ and $g(x) \neq x$, since $g$ has no fix-points. We may assume, without losing generality, that $g(v), x, g(x)$ and $g(w)$ lie on $g(\xi)$ in this order. Then

$$
\begin{aligned}
d(x, g(x)) & =d(g(v), g(x))-d(g(v), x)=d(v, x)-d(g(v), x) \\
& \leq d(v, g(v))=|g|,
\end{aligned}
$$

so $x \in \operatorname{Min}(g)$, contradicting (3.1).

Thus either the geodesics $\alpha, \beta, \xi, g(\xi)$ are pairwise disjoint off the endpoints (Figure 3.1(b)), or $\alpha$ and $\beta$ have nonempty intersection (Figure 3.1(a)). First consider the case where $\alpha$ and $\beta$ can be chosen so that $\alpha \cap \beta \neq \emptyset$ and let the intersection be maximal. The intersection is a geodesic with endpoints $p$ and $q$. Decompose $\alpha=\alpha^{\prime} \cup[p, q] \cup \alpha^{\prime \prime}$ and $\beta=\beta^{\prime} \cup[p, q] \cup \beta^{\prime \prime}$. Let $S^{\prime}$ : $\triangle^{\prime} \rightarrow X$ be a minimal surface spanning the cycle $\xi * \beta^{\prime} *\left(\alpha^{\prime}\right)^{-1}$ and $S^{\prime \prime}: \triangle^{\prime \prime} \rightarrow X$ a minimal surface spanning the cycle $g(\xi) *\left(\beta^{\prime \prime}\right)^{-1} * \alpha^{\prime \prime}$ (Figure 3.1(a)).

(a)

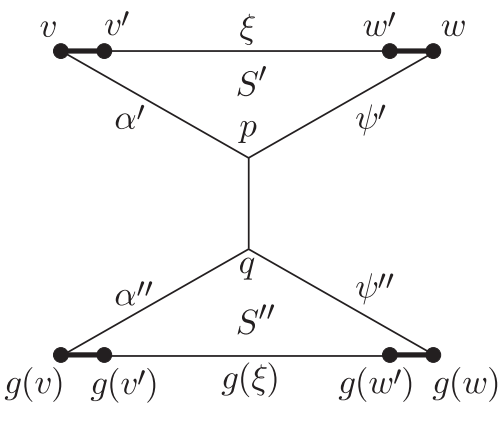

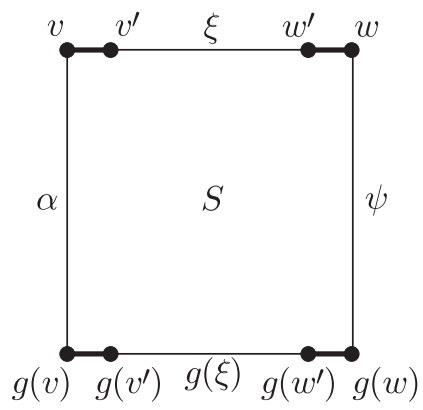

Fig. 3.1

Choose $\alpha, \beta, \xi$ such that the area of $\triangle^{\prime}$ is minimal, and among such triples choose the one minimizing the area of $\triangle^{\prime \prime}$. Thus the boundary vertices 
of $\triangle^{\prime}$ different from $v, w, p$ have nonpositive defects, so by the GaussBonnet Lemma $\operatorname{def}_{\triangle^{\prime}}(v)=\operatorname{def}_{\triangle^{\prime}}(w)=\operatorname{def}_{\triangle^{\prime}}(p)=2$ and the defects at all other vertices of $\triangle^{\prime}$ are equal to 0 . A similar calculation proves that either $\operatorname{def}_{\triangle^{\prime \prime}}(g(v))=2$ or $\operatorname{def}_{\triangle^{\prime \prime}}(g(w))=2$ (the defects at the vertices on $\alpha^{\prime \prime}$ and $\beta^{\prime \prime}$ different from the endpoints are nonpositive and the sum of the defects along $g(\xi)$ does not exceed 1 by Fact 2.5). Thus, if we denote by $v^{\prime}$ and $w^{\prime}$ the vertices on $\xi$ neighbouring to $v$ and $w$, respectively, as in Figure 3.1 (we allow $\left.v^{\prime}=w^{\prime}\right)$, then either $v^{\prime}$ to $g\left(v^{\prime}\right)$ or $w^{\prime}$ to $g\left(w^{\prime}\right)$ can be connected by a polygonal path of length $d(v, g(v))=d(w, g(w))=|g|$, contradicting (3.1).

Now assume we cannot choose $\alpha$ and $\beta$ with a nonempty intersection. Denote by $S: \triangle \rightarrow X$ a minimal surface spanning the cycle $\xi * \beta * g(\xi)^{-1} * \alpha^{-1}$ and choose $\alpha, \beta$ and $\xi$ minimizing the area of $\triangle$ (Figure 3.1(b)). Thus, the defects at the vertices on $\alpha$ and $\beta$ different from their endpoints are nonpositive. Hence, the sum of the defects along $\alpha$ plus the defects at the endpoints of $\alpha$ cannot exceed 2, as otherwise either $\operatorname{def}(v)=\operatorname{def}(g(v))=2$ or $\operatorname{def}(v)=2, \operatorname{def}(g(v))=1$ (or conversely) and the defect at any vertex on $\alpha$ different from its endpoints is 0 , which in both cases leads us to $d\left(v^{\prime}, g\left(v^{\prime}\right)\right) \leq$ $d(v, g(v))=|g|$, contradicting (3.1). Similarly, the sum of the defects along $\beta$ plus the defects at the endpoints of $\beta$ does not exceed 2 .

As by the Gauss-Bonnet Lemma the sum of the defects at the vertices on $\partial \triangle$ is at least 6 , it follows that there is a vertex $x \in \xi$ different from the endpoints such that $\operatorname{def}(x)+\operatorname{def}(g(x))>0$. As $\xi$ and $g(\xi)$ are geodesic, we have $\operatorname{def}(x) \leq 1$ and $\operatorname{def}(g(x)) \leq 1$, so at least one of the defects (say $\operatorname{def}(x))$ is equal to 1 . By modifying the boundary geodesics $\xi$ and $g(\xi)$ by cutting off $\triangle$ both triangles adjacent to $x$ and gluing their $g$-images at $g(x)$ we obtain a surface $S^{\prime}: \triangle^{\prime} \rightarrow X$, where $\triangle^{\prime}$ has the same area as $\triangle$, but $S^{\prime}$ is not minimal $\left(x \notin \operatorname{Min}(g)\right.$ implies $d(x, g(x))>1$, so $g(x) \in \triangle^{\prime}$ is an internal vertex adjacent to fewer than six triangles). This contradicts the minimality of the area of $\triangle$.

Hence the 1-skeleton of $\operatorname{Min}(g)$ is isometrically embedded in $X$.

Proposition 3.4. If $Y \subset X$ is a full subcomplex of a systolic complex $X$ such that $Y^{(1)}$ is isometrically embedded in $X$, then $Y$ is a systolic complex. In particular, for any nonelliptic simplicial isometry $g$ of $X$ the subcomplex $\operatorname{Min}(g)$ is systolic.

Proof. The complex $Y$ is 6-large (as a full subcomplex of a systolic complex) and connected (since $Y^{(1)} \subset X$ is an isometric embedding). Thus, we only need to prove that $\pi_{1}(Y)=0$.

It suffices to prove that any loop in $Y^{(1)}$ is homotopically trivial in $Y$. Suppose that, on the contrary, there is a loop in $Y^{(1)}$ which is not contractible in $Y$, and let $\gamma$ be the shortest such loop. Then $\gamma$ is embedded, $|\gamma|>3$ (by the flagness of $Y$ ) and any subpath of $\gamma$ of length not greater than $\frac{1}{2}|\gamma|$ is 
geodesic (as otherwise there would be vertices $v, w \in \gamma$ disconnecting $\gamma$ into subpaths $\gamma_{1}$ and $\gamma_{2}$ so that for a geodesic $\xi$ connecting $v$ to $w$ the loops $\gamma_{1} \cup \xi$ and $\gamma_{2} \cup \xi$ would be homotopically trivial by the minimality of $|\gamma|$ ). Thus, the loop $\gamma$ can be covered by not more than five geodesic subpaths $\gamma_{1}, \ldots, \gamma_{5}$ so that every vertex of $\gamma$ occurs as an internal vertex of $\gamma_{i}$ for precisely one $i$. It follows that for a minimal surface $S: \triangle \rightarrow X$ spanning $\gamma$, the sum of the defects at the vertices of $\partial \triangle$ is at most 5 by Fact 2.5, contradicting the Gauss-Bonnet Lemma. Therefore, $\pi_{1}(Y)=0$.

The last part of the proposition follows from Proposition 3.3.

\subsection{An invariant geodesic}

THEOREM 3.5. Let $g$ be a nonelliptic simplicial isometry of a uniformly locally finite systolic complex $X$. Then there is a $g^{n}$-invariant geodesic for some $n \geq 1$.

Proof. By Propositions 3.3 and 3.4 we may assume, without losing generality, that $\operatorname{Min}(g)=X$. Denote by $G$ the cyclic group of isometries generated by $g$. Since $g^{n}$ is nonelliptic for any $n \geq 1$ (Proposition 3.1), the action of $G$ on $X$ is free.

CASE 1. The action of $G$ on $X=\operatorname{Min}(g)$ is not cocompact and $|g|>3$.

Choose vertices $v_{1}, v_{2} \in X$ such that the $(|g|+1)$-neighbourhoods of the orbits $G v_{1}$ and $G v_{2}$ are disjoint. Connect $v_{1}$ to $g\left(v_{1}\right)$ and $v_{2}$ to $g\left(v_{2}\right)$ by geodesics $\alpha_{1}$ and $\alpha_{2}$, respectively. Let $\beta$ be a geodesic connecting $v_{1}$ to $v_{2}$. By Lemma 2.3 there exists a simplicial map $p: \triangle \rightarrow X$, where $\triangle$ is a triangulation of a disc, such that the restriction of $p$ to $\partial \triangle$ is the closed path which is the concatenation $\alpha_{1} * g(\beta) * \alpha_{2}^{-1} * \beta^{-1}$. Since $|g|>3$, the quotient space $X / G$ is a simplicial locally 6-large complex and we obtain as the quotient of $p$ a simplicial map $f: A \rightarrow X / G$, where $A$ is a triangulation of an annulus. Now we modify $A$ and $f$ applying four types of operations:

(a) If there exists in $A$ a cycle $\xi$ of length 3 not bounding a triangle of $A$, then by the assumption $|g|>3$ and the flagness of $X / G$ the cycle $\xi$ is homotopically trivial, so it disconnects $A$ into two components, one of them being a triangulation of a disc. By replacing this component with a single triangle we obtain another triangulation of an annulus.

(b) If any cycle of length 3 in $A$ bounds a triangle and there is an internal vertex $v \in A$ adjacent to four or five triangles, we cut out the open star of $v$ and glue in the disc without internal vertices instead (this is possible, since $X / G$ is locally 6-large), obtaining another simplicial triangulation of an annulus.

(c) If any cycle of length 3 in $A$ bounds a triangle and there exists an internal vertex $v \in A$ such that $f(\partial N(v))$ bounds a disc having no internal vertices, then we proceed as in (b) with the open star of $v$. 
(d) If we cannot apply the procedure from (a) or (b) and there exist two internal vertices $v, w \in A$ connected by an edge such that $f(\partial N(v w))$ bounds a disc with at most one internal vertex, then we cut out the interior of $N(v w)$ and glue in such a filling.

As we modify $A$, we modify $f$. Since each operation lowers the number of vertices in $A$, the procedure terminates. Thus we obtain a map $f^{\prime}: A^{\prime} \rightarrow$ $X / G$, where $A^{\prime}$ is a triangulation of an annulus for which every internal vertex is adjacent to at least six triangles and $f^{\prime}$ satisfies condition (v) of Theorem 2.6. The boundary $\partial A^{\prime}$ is the disjoint union of two circles: $c_{1}$ and $c_{2}$, each of length $|g|$.

Let $\widetilde{f}^{\prime}: \widetilde{A}^{\prime} \rightarrow X$ be the universal covering of $f^{\prime}$, where $\widetilde{A}^{\prime}$ is a triangulation of a strip $(\widetilde{X / G}=X$, since by [JS2, Theorem 4.1] systolic complexes are contractible). By Fact 3.2 the paths $\widetilde{f}^{\prime}\left(\widetilde{c}_{1}\right)$ and $\widetilde{f}^{\prime}\left(\widetilde{c}_{2}\right)$ are $|g|$-geodesics in $X$, so there are no vertices of defect 2 on $\partial A^{\prime}$, and every arc in $\partial A^{\prime}$ with both endpoints at vertices of defects 1 contains a vertex of negative defect. Thus the sum of the defects at the boundary vertices of $A^{\prime}$ is nonpositive and by the construction of $A^{\prime}$ every internal vertex has a nonpositive defect. Since the Euler characteristic of an annulus is 0, the combinatorial Gauss-Bonnet Theorem implies that every internal vertex of $A^{\prime}$ has defect 0 and the sum of the defects at the boundary vertices is equal to 0 . Hence there are no vertices on $\partial A^{\prime}$ of defects lower than -1 and any two vertices on $\partial A^{\prime}$ of defects -1 are separated by a vertex of defect 1 .

Thus, the strip $\widetilde{A}^{\prime}$ satisfies conditions (i)-(iii) of Theorem 2.6. Condition (iv) follows from the fact that

$$
\operatorname{dist}_{X}\left(\widetilde{f}^{\prime}\left(\widetilde{c}_{1}\right), \widetilde{f}^{\prime}\left(\widetilde{c}_{2}\right)\right) \geq \operatorname{dist}_{X}\left(\mathcal{N}_{|g|}\left(G v_{1}\right), \mathcal{N}_{|g|}\left(G v_{2}\right)\right)>3 .
$$

Hence, by Theorem 2.6 any boundary component of the strip is mapped to a geodesic in $X$ and by the construction of the strip it is a $g$-invariant geodesic.

CASE 2. The action of $G$ on $X=\operatorname{Min}(g)$ is not cocompact and $|g| \leq 3$.

Consider the sequence of subcomplexes

$$
X_{0}=X, \quad X_{n}=\operatorname{Min}_{X_{n-1}}\left(g^{n}\right), \quad \text { for } n=1,2, \ldots
$$

By Propositions 3.3 and 3.4 the subcomplex $X_{n} \subset X_{n-1}$ is systolic, $g$ invariant and isometrically embedded. Since for any $n$ the complex $X_{n}$ is uniformly locally finite, $G$ acts freely on $X_{n}$ and we have

$$
d\left(v, g^{k}(v)\right)=\left|g^{k}\right|_{X_{n}} \quad \text { for } k=1, \ldots, n \text { and } v \in X_{n}^{(0)},
$$

there exist $n$ and $k \leq n$ such that $\left|g^{k}\right|_{X_{n}}>3$. As in Case 1, we obtain a $g^{k}$-invariant geodesic $l$ in $X_{n}$. As $X_{n} \subset X$ is a $g$-invariant and isometrically embedded subcomplex, $l$ is a $g^{k}$-invariant geodesic in $X$.

CASE 3. The action of $G$ on $X=\operatorname{Min}(g)$ is cocompact. 
Since any ball in $X$ contains a finite number of vertices (by the local finiteness of $X$ ) and $\operatorname{diam}(X)=\infty$ (the action of $G \cong \mathbb{Z}$ is free), there exists (by a standard diagonal argument) a bi-infinite geodesic $l$ in $X$.

As $G \cong \mathbb{Z}$ acts freely cocompactly on $X$, the space $X$ has two ends. Thus there exists a finite subcomplex $B \subset X$ disconnecting $X$ such that any bi-infinite geodesic in $X$ intersects $B$. Let $n$ be the number of vertices in $B$. Then for any $i$ there are two geodesics among $l, g(l), \ldots, g^{n}(l)$ with a common vertex in $g^{i}(B)$. Hence there are geodesics $g^{j}(l)$ and $g^{k}(l)$ with an infinite intersection for some $0 \leq j<k \leq n$. The existence of a $g^{k-j}$-invariant geodesic follows from the subsequent lemma.

LEMMA 3.6. Let $f$ be a simplicial isometry without fix-points of a locally finite simplicial complex $X$, and $\gamma$ a geodesic in $X$. If $\gamma \cap f(\gamma)$ contains infinitely many vertices, then there is an $f$-invariant geodesic in $X$.

Proof. We construct inductively a sequence of geodesics $\gamma_{i}, i=0,1,2, \ldots$, such that $\gamma_{i} \cap f\left(\gamma_{i}\right)$ has infinitely many vertices and contains a geodesic of length at least $i$ and contains $a_{0}$. Put $\gamma_{0}:=\gamma$. Suppose we have already constructed $\gamma_{i}$ and $\left[a_{i}, b_{i}\right] \subset \gamma_{i} \cap f\left(\gamma_{i}\right)$ is a maximal geodesic in the intersection. We may assume, not losing generality, that $b_{i}$ separates $f\left(b_{i}\right)$ and $a_{i}$ on $f\left(\gamma_{i}\right)$. Then $a_{i}$ separates $b_{i}$ and $f^{-1}\left(a_{i}\right)$ on $\gamma_{i}$. Since $\gamma_{i}$ and $f\left(\gamma_{i}\right)$ have infinite intersection, there is $x_{i} \in \gamma_{i} \cap f\left(\gamma_{i}\right)$ such that either $f\left(b_{i}\right)$ separates $b_{i}$ and $x_{i}$ on $f\left(\gamma_{i}\right)$, or $f^{-1}\left(a_{i}\right)$ separates $a_{i}$ and $x_{i}$ on $\gamma_{i}$. In the first case we obtain $\gamma_{i+1}$ from $\gamma_{i}$ by replacing the segment with endpoints $b_{i}$ and $x_{i}$ with the segment of $f\left(\gamma_{i}\right)$ with the same endpoints. Then there is a common segment $\left[a_{i}, f\left(b_{i}\right)\right] \subset \gamma_{i+1} \cap f\left(\gamma_{i+1}\right)$. In the second case we define $f\left(\gamma_{i+1}\right)$ to be $f\left(\gamma_{i}\right)$ with the segment with endpoints $x_{i}$ and $a_{i}$ replaced with the segment of $\gamma_{i}$ having the same endpoints. Then there is a common segment $\left[f^{-1}\left(a_{i}\right), b_{i}\right] \subset \gamma_{i+1} \cap f\left(\gamma_{i+1}\right)$.

Now fix an arbitrary vertex $v \in X$. By the local finiteness of $X$ and a standard diagonal argument we can choose a subsequence of geodesics $\gamma_{i}^{\prime}$ such that the sequences $\gamma_{i}^{\prime}$ and $f\left(\gamma_{i}^{\prime}\right)$ are convergent (uniformly on compact sets). Hence the limiting geodesics $\bar{\gamma}$ and $f(\bar{\gamma})$ have some common infinite ray.

Finally, either the sequence $f^{n}(\bar{\gamma}), n=1,2, \ldots$, or the sequence $f^{-n}(\bar{\gamma})$, $n=1,2, \ldots$, converges to an $f$-invariant geodesic.

REMARK. If there exists a $g^{n}$-invariant geodesic in $X$, then for any vertex $x \in \operatorname{Min}\left(g^{n}\right) \subset X$ there exists a $g^{n}$-invariant geodesic passing through $x$.

Proof. We construct a polygonal $g^{n}$-invariant line $l$ passing through $x$ as in Fact 3.2. By the existence of a $g^{n}$-invariant geodesic and by the fact that $l \subset \operatorname{Min}\left(g^{n}\right)$, the triangle inequality implies that $l$ is also a geodesic. 
3.3. Thick axis Fix some integer $k \geq 2$. Recall that $A_{k}$ is a simplicial complex with $A_{k}^{(0)}=\mathbb{Z}$ such that $\sigma \subset \mathbb{Z}$ spans a simplex if and only if $\left|a-a^{\prime}\right| \leq k$ for all $a, a^{\prime} \in \sigma$. A thick geodesic in a systolic complex $X$ will be a full subcomplex $A_{k} \subset X, k \geq 1$, such that for any $a, a^{\prime} \in \mathbb{Z}$ we have

$$
k \mid a-a^{\prime} \Rightarrow d_{X}\left(a, a^{\prime}\right)=d_{A_{k}}\left(a, a^{\prime}\right) .
$$

FACT 3.7. Let $A_{k}, k \geq 1$, be a thick geodesic in a systolic complex $X$. Then:

(1) There is an ordinary geodesic in $X$ at Hausdorff distance at most 1 from $A_{k}$.

(2) The restriction to $A_{k}$ of a nonelliptic isometry of $X$ stabilizing $A_{k}$ has the form $\gamma_{n}: x \mapsto x+n$ for some $n \in \mathbb{Z}$.

Proof. Part (1) follows from the fact that the vertices $j k \in A_{k}, j \in \mathbb{Z}$, span a geodesic in $A_{k}$, hence in $X$. Part (2) is implied by the fact that if the number $\left|a-a^{\prime}\right|$ for $a, a^{\prime} \in A_{k}^{(0)}$ is not greater than $k$, then it can be described as $\operatorname{dim}(\bigcap \sigma)$ where the intersection is taken over all simplices $\sigma$ in $A_{k}$ such that $\operatorname{dim} \sigma=k$ and $a, a^{\prime} \in \sigma$.

THEOREM 3.8. Let $g$ be a simplicial isometry of a uniformly locally finite systolic complex $X$. Then either there is a g-invariant simplex (elliptic case) or there is a g-invariant thick geodesic (hyperbolic case).

Proof. By Theorem 3.5, if $g$ is nonelliptic, then there exists a $g^{n}$-invariant geodesic in $X$ for some $n \geq 1$. Let $n$ be minimal. If $n=1$ then there is an ordinary $g$-invariant geodesic in $X$ (isomorphic to $A_{1}$ ), thus suppose $n>1$.

Denote by $\mathbb{E}_{\triangle}^{2}$ a triangulation of the Euclidean plane by congruent equilateral triangles.

STEP 1. There exist translations $\tau, \sigma \in \operatorname{Isom}\left(\mathbb{E}_{\triangle}^{2}\right)$, a $\tau$-invariant geodesic $m \subset \mathbb{E}_{\triangle}^{2}$ and a simplicial map $F: \mathbb{E}_{\triangle}^{2} \rightarrow X$ such that:

(i) $\tau$ and $\sigma$ generate a group $T \cong \mathbb{Z}^{2}$ of simplicial isometries of $\mathbb{E}_{\triangle}^{2}$;

(ii) $F \circ \tau=g^{n} \circ F$ and $F \circ \sigma=g \circ F$;

(iii) $F(m) \subset X$ is a $g^{n}$-invariant geodesic.

Let $l$ be a $g^{n}$-invariant geodesic in $X$ and define $l^{\prime}=g(l)$. The geodesics $l$ and $l^{\prime}$ are disjoint by the minimality of $n$ and by Lemma 3.6. Choose vertices $x \in l, x^{\prime} \in l^{\prime}$ and join them by a geodesic $\gamma$ in $X$. Denote by $\alpha$ and $\alpha^{\prime}$ the segments of $l$ and $l^{\prime}$ connecting $x$ to $g^{n}(x)$ and $x^{\prime}$ to $g^{n}\left(x^{\prime}\right)$, respectively. Let $f: \triangle \rightarrow X$ be a simplicial map (a minimal surface), where $\triangle$ is a simplicial disc, such that the restriction of $f$ to $\partial \triangle$ coincides with the closed path $\alpha * g^{n}(\gamma) *\left(\alpha^{\prime}\right)^{-1} * \gamma^{-1}$ (such a map exists by Lemma 2.3). Let $l, x, x^{\prime}$ and $\gamma$ be chosen so that the area of $\triangle$ is minimal. 
By gluing the maps $\left(g^{n}\right)^{i} \circ f$ for $i \in \mathbb{Z}$ we obtain a $g^{n}$-equivariant simplicial map $f^{\prime}: S \rightarrow X$, where $S$ is a triangulation of a strip $\mathbb{R} \times I$ on which $g^{n}$ acts by translation and each component of $\partial S$ is mapped to a geodesic in $X$. By the systolicity of $X$ every internal vertex of $S$ is adjacent to at least six triangles (for internal vertices of $\triangle \subset S$ this comes from Lemma 2.3, for vertices on $\gamma$ it comes from the choice of $\gamma$ minimizing the area of $\triangle$ ).

Denote the boundary components of $S$ by $m$ and $m^{\prime}$ (so that $f^{\prime}(m)=l$ and $\left.f^{\prime}\left(m^{\prime}\right)=l^{\prime}\right)$ and define $p: m \rightarrow m^{\prime}$ to be the isomorphism satisfying $f^{\prime} \circ p=\left.g \circ f^{\prime}\right|_{m}$.

We prove that $\operatorname{def}(x)+\operatorname{def}(p(x)) \leq 0$ for all vertices $x \in m$. This is immediate in the case where $\left|g^{n}\right|=1$, as then by the $g^{n}$-invariance and the geodesity of $m$ and $m^{\prime}$, any boundary vertex $x \in \partial S$ has a nonpositive defect. Thus assume $\left|g^{n}\right|>1$. Since $m$ and $m^{\prime}$ are geodesics in $S$, there are no vertices on $\partial S$ of defect 2 . Consider a vertex $x \in m \subset S$ of defect 1 . If the defect at $p(x) \in m^{\prime} \subset S$ is nonnegative, then we can modify $S$ by cutting out the triangles adjacent to the vertices $\left(g^{n}\right)^{i}(x) \in m \subset S$ (the open stars of these vertices are disjoint, as $\left.\left|g^{n}\right|>1\right)$ for $i \in \mathbb{Z}$ and gluing their $g$-images at the vertices $p\left(\left(g^{n}\right)^{i}(x)\right) \in m^{\prime} \subset S$, so that the modified map $\bar{f}: \bar{S} \rightarrow X$ is $g^{n}$-equivariant and maps $\partial \bar{S}$ to the disjoint union of two geodesics $\bar{l}$ and $g(\bar{l})$. If the distance between $p(x)$ and the set $\left\{\left(g^{n}\right)^{i}(x): i \in \mathbb{Z}\right\}$ is greater than 1 , then $\bar{S}$ contains an internal vertex adjacent to fewer than six triangles, which contradicts the minimality of the area of $\triangle$ (by the systolicity of $X)$. Otherwise, $\left|g^{1-n i}\right|=1$ for some $i \in \mathbb{Z}$, so $l$ and $g(l)$ are Hausdorff 1-close and disjoint, which implies $\operatorname{def}(x)+\operatorname{def}(p(x)) \leq 0$ for all vertices $x \in m$, completing the proof of this inequality.

Now by the Gauss-Bonnet Lemma applied to the subcomplexes of $S$ bounded by long enough subsegments of $m$ and $m^{\prime}$ and two geodesics joining the endpoints of these subsegments, we obtain $\operatorname{def}(x)+\operatorname{def}(p(x))=0$ for all vertices $x \in m$, and any internal vertex $v \in S$ is adjacent to exactly six triangles (see Fact 2.5). Therefore, by gluing the maps $g^{i} \circ f^{\prime}$ for $i \in \mathbb{Z}$, we obtain a simplicial map $F: \mathbb{E}_{\triangle}^{2} \rightarrow X$ satisfying (i)-(iii), where $\sigma$ is determined by $p$.

SteP 2. Choose $\tau, \sigma, m$ and $F$ satisfying conditions (i)-(iii) of Step 1 so that the number of orbits of the action of $T$ on the 0 -skeleton of $\mathbb{E}_{\triangle}^{2}$ is minimal. Then $m$ and $\sigma(m)$ are Hausdorff 1-close.

Since the $\tau$-invariant geodesic $m \subset \mathbb{E}_{\triangle}^{2}$ is mapped by $F$ to a $g^{n}$-invariant geodesic in $X$, any $\tau$-invariant geodesic $\bar{m} \subset \mathbb{E}_{\triangle}^{2}$ is mapped to a $g^{n}$-invariant geodesic in $X$. This comes from the following inequality, satisfied for all $x \in m, \bar{x} \in \bar{m}$ and $N, i \in \mathbb{Z}$ :

$$
N d\left(F(\bar{x}), F\left(\tau^{i}(\bar{x})\right)\right) \geq N d\left(F(x), F\left(\tau^{i}(x)\right)\right)-2 d(F(x), F(\bar{x})) .
$$


If $\left.F\right|_{N(v)}$ were an isometric embedding for every vertex $v \in \mathbb{E}_{\triangle}^{2}$, then by Theorem 2.7 the map $F$ would be an isometric embedding. However, $F$ is not injective, hence there is a vertex $v \in \mathbb{E}_{\triangle}^{2}$ such that $\left.F\right|_{N(v)}$ is not an isometric embedding (so by the systolicity of $X$ the closed path $F(\partial N(v)$ ) can be filled with a 2-disc without internal vertices). If the distance between $m$ and $\sigma(m)$ is greater than 1 , then there is a $\tau$-invariant geodesic $\bar{m}$ disjoint from the orbit $T v$. Thus, by repeating the procedure of Step 1 starting with $\bar{l}=F(\bar{m})$ instead of $l=F(m)$ we obtain a contradiction with the minimality of the number of $T$-orbits. Therefore $m$ and $\sigma(m)$ are Hausdorff 1-close.

SteP 3. If $\tau, \sigma, m$ and $F$ are chosen as in Step 2, then $m \subset \mathbb{E}_{\triangle}^{2}$ is a convex geodesic.

We introduce a coordinate system on $\mathbb{E}_{\triangle}^{2}$ by choosing a vertex $0 \in m \subset$ $\mathbb{E}_{\triangle}^{2}$ and a triangle adjacent to 0 . The edges of the triangle containing the vertex 0 represent base vectors $e_{1}$ and $e_{2}$. Let the coordinates of the vectors $\overrightarrow{0 \tau(0)}$ and $\overrightarrow{0 \sigma(0)}$ be $(\alpha, \beta)$ and $(a, b)$, respectively, $\alpha, \beta, a, b \in \mathbb{Z}$. We may choose $e_{1}$ and $e_{2}$ so that $\alpha, \beta \geq 0$.

We have to prove that either $\alpha=0$ or $\beta=0$. Assume, on the contrary, that $\alpha, \beta>0$. Then for any vertices $p_{i}=\left(x_{i}, y_{i}\right) \in \mathbb{E}_{\triangle}^{2}, i=0,1$, we have

$$
x_{0} \leq x_{1} \wedge y_{0} \leq y_{1} \Rightarrow d_{\mathbb{E}_{\triangle}^{2}}\left(p_{0}, p_{1}\right)=d_{X}\left(F\left(p_{0}\right), F\left(p_{1}\right)\right),
$$

since there exist integers $j<k$ such that $p_{0}$ and $p_{1}$ lie in this order on a geodesic joining $\tau^{j}(0)$ and $\tau^{k}(0)$, and the latter vertices lie on the geodesic $m$ which is mapped to a geodesic in $X$.

If $a, b \geq 0$ or $a, b \leq 0$, then by (3.2) a geodesic in $\mathbb{E}_{\triangle}^{2}$ passing through $\sigma^{k}(0)=(k a, k b)$ for all $k \in \mathbb{Z}$ is mapped by $F$ to a geodesic $\gamma$ in $X$ and $\gamma \cap g(\gamma)$ has infinitely many vertices (since $F \circ \sigma=g \circ F$ ). By Lemma 3.6 there is a $g$-invariant geodesic in $X$, contrary to the assumption.

The only case left is when $a$ and $b$ are of different signs. Without loss of generality we can assume $a<0<b$. The set of vertices of any geodesic joining 0 and $\tau(0)=(\alpha, \beta)$ is contained in

$$
\{(x, y): 0 \leq x \leq \alpha, 0 \leq y \leq \beta, x, y \in \mathbb{Z}\} .
$$

Thus the geodesic $m$ passing through $\tau^{k}(0)=(k \alpha, k \beta)$ for all $k \in \mathbb{Z}$ is contained in

$$
P=\{(x, y): k \alpha \leq x \leq(k+1) \alpha, k \beta \leq y \leq(k+1) \beta, x, y, k \in \mathbb{Z}\} .
$$

Since $\sigma(m)$ is at Hausdorff distance 1 from $m$, we have $\operatorname{dist}(\sigma(0), P) \leq 1$. There are only six vectors of length 1 in $\mathbb{E}_{\triangle}^{2}: \pm e_{1}, \pm e_{2}, e_{1}-e_{2}, e_{2}-e_{1}$, hence the coordinates of $\sigma(0)=(a, b)$ satisfy: $a=-1$ and $1 \leq b \leq \beta+1$ or $b=1$ and $-\alpha-1 \leq a \leq-1$ (since $a<0<b$ ). 
In the first case we prove by induction that $m$ passes through $(0, k(b-1))$ for any $k \in \mathbb{N}$ : if the geodesic $m$ passes through $(0, j(b-1))$ for some $j \geq 0$, then

$$
\sigma((0, j(b-1)))=(-1,(j+1)(b-1)+1)
$$

is at distance 1 to $m$, which implies that $m$ passes through $(0,(j+1)(b-1))$. In the second case we similarly deduce that $m$ passes through $(k(a+1), 0)$ for any $k \in \mathbb{N}$. Both results contradict the assumption $\alpha, \beta>0$.

Now we define a thin strip to be the infinite simplicial complex $S$ spanned by two convex geodesics in $\mathbb{E}_{\triangle}^{2}$ at distance 1 to each other (as in Figure 3.2). We are especially interested in simplicial maps $f: S \rightarrow X$ with thin strip $S$ such that the images of the boundary geodesics of $S$ are the geodesics $g^{i}(l)$ and $g^{j}(l)$ for $0 \leq i<j<n$. Since $g^{i}(l)$ and $g^{j}(l)$ are disjoint (otherwise by Lemma 3.6 we obtain a $g^{j-i}$-invariant geodesic, contrary to the minimality of $n$ ), the map $f$ is injective, so we may assume $S$ is a subcomplex of $X$. If such a map $f$ exists, we say that $g^{i}(l)$ and $g^{j}(l)$ span a thin strip. In Steps 2 and 3 we proved that $l$ and $g(l)$ span a thin strip.

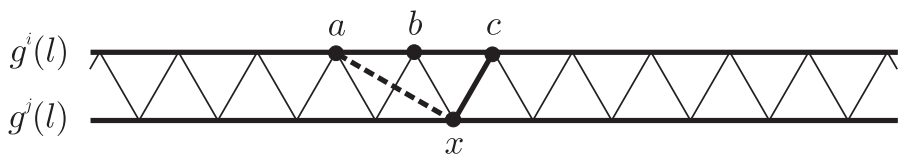

Fig. 3.2

Let $g^{i}(l)$ and $g^{j}(l), 0 \leq i<j<n$, span a thin strip $S$. The subcomplex $S^{\prime} \subset S$ consisting of all edges $e \subset S$ such that $e \not \subset \partial S$ is combinatorially equivalent to a line. Thus, it determines two linear orders on the set of vertices of $g^{i}(l) \cup g^{j}(l)$. We define $\prec_{i j}$ to be the order for which $x \prec g^{n}(x)$ for some (hence every) $x \in g^{i}(l) \cup g^{j}(l)$.

STEP 4. If the geodesics $g^{i}(l)$ and $g^{j}(l), 0 \leq i<j<n$, span a thin strip $S \subset X$, then $S$ is a full subcomplex of $X$.

Since the boundary lines of $S$ are geodesic and disjoint (Lemma 3.6), $S \subset X$ is not a full subcomplex if and only if there are vertices $a$ and $x$ in different components of $\partial S$ such that $d_{S}(a, x)=2$ and $d_{X}(a, x)=1$ (as in Figure 3.2). Suppose this is the case.

The boundary geodesics $g^{i}(l)$ and $g^{j}(l)$ are $g^{n}$-invariant and $g^{n}$ acts on them as a translation of length $\xi \geq 1$. Thus $g^{2 n}$ acts as a translation of length $2 \xi \geq 2$. If we replace the vertices $g^{2 \alpha n}(b) \in g^{i}(l)$ with $g^{2 \alpha n}(x) \in g^{j}(l)$ for all $\alpha \in \mathbb{Z}$ we obtain a $g^{2 n}$-invariant geodesic $l^{\prime}$. Since $x \in g^{j}(l)$, we have $g^{i-j}(x) \in g^{i}(l)$ and $g^{i-j+n}(x) \in g^{i}(l)$. The vertices $g^{i-j}(x)$ and $g^{i-j+n}(x)$ are not in the same $g^{2 n}$-orbit. As $g^{i}(l) \backslash l^{\prime}$ contains only one $g^{2 n}$-orbit, $l^{\prime}$ passes either through $x$ and $g^{i-j}(x)$ or through $x$ and $g^{i-j+n}(x)$. By Lemma 3.6 
there exists a $g^{j-i}$-invariant geodesic or $g^{i-j+n}$-invariant geodesic, contradicting the minimality of $n$.

STEP 5. If $g^{i}(l)$ and $g^{j}(l)$ span a thin strip and so do $g^{j}(l)$ and $g^{k}(l)$ for $0 \leq i<j<k<n$, then $g^{i}(l)$ and $g^{k}(l)$ span a thin strip. Moreover, the relation $\prec_{i j k}=\prec_{i j} \cup \prec_{j k} \cup \prec_{i k}$ is transitive.

Denote the strips spanned by $g^{i}(l), g^{j}(l)$ and by $g^{j}(l), g^{k}(l)$ by $S$ and $S^{\prime}$, respectively. By gluing the strips $g^{\alpha(k-i)}(S)$ and $g^{\alpha(k-i)}\left(S^{\prime}\right)$ for $\alpha \in \mathbb{Z}$ we obtain a simplicial map $P: \mathbb{E}_{\triangle}^{2} \rightarrow X$ (as in Figure 3.3), which is not injective, as $S=g^{n(k-i)}(S)$.

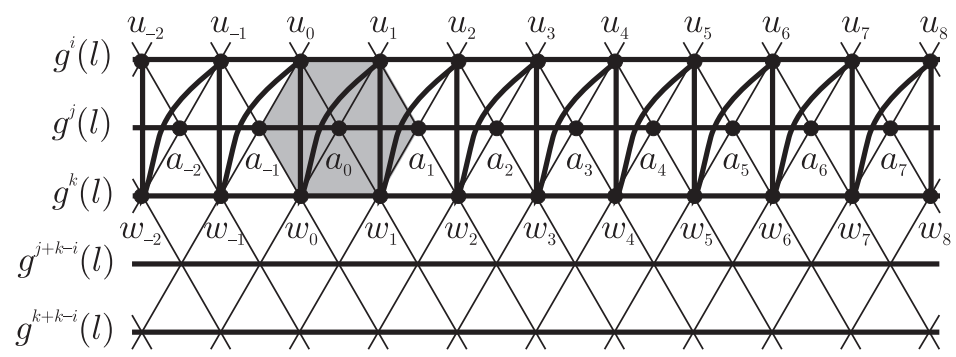

Fig. 3.3

If the restriction of $P$ to $N(v)$ were an isometric embedding for every vertex $v \in \mathbb{E}_{\triangle}^{2}$, then by Theorem $2.7, P$ would be an isometric embedding, contrary to the fact it is not injective. Thus there is a vertex $v \in \mathbb{E}_{\triangle}^{2}$ such that $\left.P\right|_{N(v)}$ is not an isometric embedding. Without loss of generality we may assume $v=a_{0} \in g^{j}(l)$ as in Figure 3.3.

The map $\left.P\right|_{N\left(a_{0}\right)}$ is injective, since $\left.P\right|_{S}$ and $\left.P\right|_{S^{\prime}}$ are injective and the geodesics $g^{i}(l)$ and $g^{k}(l)$ are disjoint (Lemma 3.6). Since $\left.P\right|_{N\left(a_{0}\right)}$ is not an isometric embedding, there are two vertices in $\partial N\left(a_{0}\right)$ not connected by an edge in $\mathbb{E}_{\triangle}^{2}$, whose images are connected by an edge. Thus, by the systolicity of $X$, the image of $\partial N\left(a_{0}\right)$ bounds a disc of area 4 . Since by Step 4 the vertex $P\left(a_{-1}\right)$ is connected by an edge neither to $P\left(u_{1}\right)$ nor to $P\left(w_{1}\right)$, and $P\left(a_{1}\right)$ is connected neither to $P\left(u_{0}\right)$ nor to $P\left(w_{0}\right)$, the filling contains the edges $P\left(u_{0}\right) P\left(w_{0}\right), P\left(u_{1}\right) P\left(w_{1}\right)$ and either $P\left(w_{0}\right) P\left(u_{1}\right)$ or $P\left(u_{0}\right) P\left(w_{1}\right)$, say the former.

By induction on $j$ we prove that the vertex $P\left(w_{j}\right) \in X$ is connected by edges to the vertices $P\left(u_{j}\right)$ and $P\left(u_{j+1}\right)$ : If we have already proved the existence of the edges $P\left(u_{j}\right) P\left(w_{j}\right)$ and $P\left(w_{j}\right) P\left(u_{j+1}\right)$, then by the systolicity the quadrilateral $P\left(w_{j}\right) P\left(u_{j+1}\right) P\left(a_{j+1}\right) P\left(w_{j+1}\right)$ has a diagonal and by Step 4 it is $P\left(u_{j+1}\right) P\left(w_{j+1}\right)$. Now again by the systolicity of $X$ the pentagon $P\left(w_{j+1}\right) P\left(u_{j+1}\right) P\left(u_{j+2}\right) P\left(a_{j+2}\right) P\left(w_{j+2}\right)$ has two diagonals: $P\left(u_{j+2}\right) P\left(w_{j+2}\right)$ and either $P\left(u_{j+1}\right) P\left(w_{j+2}\right)$ or $P\left(u_{j+2}\right) P\left(w_{j+1}\right)$. However, 
the case $P\left(u_{j+1}\right) P\left(w_{j+2}\right)$ is impossible, since then proceeding as in Step 4 we obtain a contradiction with the minimality of $n$. Thus $g^{i}(l)$ and $g^{k}(l)$ span a thin strip.

The fact that $\prec_{i j k}$ is transitive is now clear from Figure 3.3.

STEP 6. There is a thick geodesic embedded into $X$.

Define $Y=\bigcup_{i=0}^{n-1} g^{i}(l)$. By Steps 2 and 3 the geodesics $l$ and $g(l)$ span a thin strip (since $m$ is a convex geodesic in $\mathbb{E}_{\triangle}^{2}$ and $\sigma(m)$ is 1-close to $m$ ). Thus, applying Step 5 we prove by induction on $k$ that the geodesics $l$ and $g^{k}(l)$ for $0<k<n$ span a thin strip. It follows that $g^{i}(l)$ and $g^{j}(l)$ span a thin strip for $0 \leq i<j<n$. By Step 4 the subcomplex $Y \subset X$ is full.

We can now introduce on the set $Y^{(0)}$ the relation

$$
\prec=\bigcup_{0 \leq i<j<n} \prec_{i j}
$$

which by Step 5 is well-defined and transitive. It is also reflexive, antisymmetric and linear, as so are the $\prec_{i j}$. Hence $\prec$ defines a linear order on $Y^{(0)}$. Thus, we may identify $Y^{(0)}$ with $\mathbb{Z}$. Since for any two consecutive vertices $a, a^{\prime} \in g^{i}(l)$ and any $j \neq i$ there is exactly one vertex $b \in g^{j}(l)$ such that $a \prec b \prec a^{\prime}$, the vertices of $g^{i}(l)$ are identified with $k n+r_{i}, k \in \mathbb{Z}$, for some $r_{i}$. It follows that the vertices $a \in g^{i}(l)$ and $b \in g^{j}(l)$ are connected by an edge in $X$ if and only if they are identified with integers of difference not greater than $n$. Hence, the flag completion of $Y$ is a $g$-invariant thick geodesic in $X$.

COROLlary 3.9. Let $G$ be a systolic group, i.e. a group acting properly discontinuously and cocompactly on some systolic complex $X$. Then any finitely generated abelian subgroup of $G$ is undistorted.

This fact is a consequence of [JS2, Theorem 13.1], stating that systolic groups are biautomatic. Below we present an alternative proof, as an application of the theory of minimal surfaces.

Proof. As a finitely generated abelian group has a finite-index free abelian subgroup, it suffices to prove that free abelian subgroups are nondistorted. In [E, Theorem 6.1] we proved that free abelian subgroups of rank 2 of a systolic group are undistorted and that systolic groups do not contain free abelian subgroups of higher ranks.

The case $n=1$ follows directly from Theorem 3.5. Let $X$ be a systolic complex admitting a cocompact and properly discontinuous action of $G$ and let $x \in X$ be an arbitrary vertex. Then the map $g \mapsto g(x)$ defines a quasiisometry $q: G \rightarrow X$. By Theorem 3.5, for any infinite cyclic subgroup $H<G$ there exists an $H$-invariant quasi-geodesic $l$ in $X$, which is mapped by a quasi-inverse of $q$ to a quasi-geodesic $k$ in $G$, such that $k \subset G$ and 
$H \subset G$ are at finite Hausdorff distance. Thus $H \hookrightarrow G$ is a quasi-isometric embedding, i.e. $H$ is undistorted.

Acknowledgements. The author thanks Jan Dymara and Tadeusz Januszkiewicz for many helpful discussions and advice.

The author was partially supported by Polish Ministry of Science and Higher Education, MNiSW grant N201 012 32/0718.

\section{References}

[BH] M. Bridson and A. Haefliger, Metric Spaces of Non-Positive Curvature, Grundlehren Math. Wiss. 319, Springer, 1999.

[E] T. Elsner, Flats and the flat torus theorem in systolic spaces, Geom. Topology 13 (2009), 661-698.

[Gr] M. Gromov, Asymptotic invariants of infinite groups, Vol. 2 of Geometric Group Theory, G. Niblo and M. Roller (eds.), London Math. Soc. Lecture Note Ser. 182, Cambridge Univ. Press, 1993.

[Ha] F. Haglund, Complexes simpliciaux hyperboliques de grande dimension, Prépublication Orsay 71, 2003.

[HS] F. Haglund and J. Swiątkowski, Separating quasi-convex subgroups in 7-systolic groups, Groups Geom. Dynam. 2 (2008), 223-244.

[JS1] T. Januszkiewicz and J. Świątkowski, Filling invariants in systolic complexes and groups, Geom. Topology 11 (2007), 727-758.

[JS2] —, 一, Simplicial nonpositive curvature, Publ. Math. Inst. Hautes Études Sci. 104 (2006), 1-85.

Department of Mathematics

The Ohio State University

$231 \mathrm{~W} 18$ th Ave.

Columbus, OH 43210, U.S.A.
Instytut Matematyczny Uniwersytet Wrocławski pl. Grunwaldzki 2/4 50-384 Wrocław, Poland E-mail: elsner@math.uni.wroc.pl http://www.math.uni.wroc.pl/ ${ }^{\sim}$ elsner

Received 4 June 2008;

in revised form 9 February 2009 